\title{
Small angle neutron scattering from helium bubbles in metals
}

\author{
F CARSUGHI \\ Institut für Festkörperforschung, Forschungszentrum Jülich, D-52425 Jülich, Germany
}

\begin{abstract}
Small angle neutron scattering (SANS) has been widely used in investigating defects in metals, and in particular, to characterize the helium bubble population in implanted materials. The main advantage in using SANS is the non-destructive feature of the tests and the quantitative results obtained by averaging over a large sample volume. SANS is a powerful technique, very sensitive to microstructural changes and its use was of fundamental importance to show the bimodal distribution of the bubble population: in the vicinity of grain boundaries and free surface and inside grains, respectively. Here the most important applications of the SANS technique to the study of the helium bubbles in implanted materials are reviewed. Most of the work has been done on nickel samples, but also a ternary alloy $\mathrm{Fe}-\mathrm{Ni} 15 \% \mathrm{wt}-\mathrm{Cr} 15 \% \mathrm{wt}$ and a steel (MANET) with a more complicated structure have been successfully investigated. Different annealing treatments, isothermal and isochronal, were investigated in order to determine the active mechanisms of the bubble coarsening and their activation energies. From the SANS data the bubble size distributions have been determined, from which parameters such as mean radius and density of the bubble population have been calculated. The gas pressure inside the bubbles was also determined by the contrast variation technique in SANS and by a computational procedure, and an excellent agreement was found between the results. These results show a marked overpressure inside the bubbles as compared to thermodynamical equilibrium values of about $3 \mathrm{GPa}$. A comparison with results obtained by other techniques confirms the validity of SANS, which has to be considered as a complementary technique for its indirect image of the sample.
\end{abstract}

Keywords. Small angle neutron scattering; $\alpha$-implanted materials; He bubbles.

\section{Introduction}

In nuclear technology (fast breeder reactors, future fusion reactors and tritium storage) as well as in spallation sources, helium is produced, via $(n, \alpha),\left(T,{ }^{3} \mathrm{He}\right)$ and $(p, \alpha)$ reactions, respectively. In the worst case (tritium storage and spallation sources) up to 100 appm He per day can be introduced into the structural materials. Since helium is practically insoluble in solids, the atoms tend to precipitate into bubbles; the nucleation and the growth of these bubbles at high temperatures influence drastically the mechanical properties of the materials (Ullmaier 1984). The understanding of the basic processes related to the presence of helium inside the materials is therefore very important to tailor new materials and to determine the lifetime of the components. Simulation techniques, such as fission reactor irradiation or high energy $\alpha$-particles implantation (Ullmaier 1981), were widely used to produce samples for the investigations. Both simulation techniques present drawbacks but since with the latter a higher implantation rate can be achieved (up to few \% per h), it was used for the works presented here. Many different experimental methods were used to characterize the helium bubble population. (Ullmaier 1991); among them transmission electron microscopy (TEM) is the most common because of its direct imaging of the microstructure without much elaboration of the experimental results. 


\section{Small angle neutron scattering}

Small angle scattering of cold neutrons represents an excellent tool to investigate the inhomogeneities of dimensions in the $\mathrm{nm}$ and the $\mu \mathrm{m}$ range, such as for example, precipitates, voids and helium bubbles. The scattering is an elastic interaction related to the difference of the scattering length density between the scattering centres and the matrix. SANS is also very sensitive to the microstructural changes and it has been widely used to characterize the microstructure of structural material (Kostorz 1979; Price and Sköld 1986). The general theory of small angle scattering (SAS) lies outside the aim of this contribution and it can be found elsewhere (Guinier and Fournet 1955; Kostorz 1979; Price and Sköld 1986). It was developed for X-rays, and only subsequently it was applied to neutrons. The main advantage of neutrons compared to $\mathrm{X}$-rays is the low absorption, the larger beam size and that the neutron cross sections depend on the nuclear structure and not on the electronic structure; this makes possible to discriminate between different isotopes of the same element, allowing the contrast variation measurements (Stuhrmann and Kirste 1965). A SANS measurement will provide the macroscopic differential coherent scattering cross section $d \Sigma / d \Omega$ as a function of the exchanged wave vector $\vec{Q}$. Under the following assumptions:

- presence of two homogeneous phases of constant chemical composition and density;

- randomly oriented scattering centres;

- dilute system, with volume fraction up to few \%;

the SANS theory can be extremely simplified. In case where all the scattering centres have not the same dimension, the SANS cross section can be written as

$$
\frac{\mathrm{d} \Sigma}{\mathrm{d} \Omega}(Q)=(\Delta \rho)^{2} \int N(R) V^{2}(R)|F(Q, R)|^{2} \mathrm{~d} R,
$$

where $\Delta \rho$ is the difference of the scattering length density (contrast factor), $N(R)$ the size distribution function (SDF), $V(R)$ and $F(Q, R)$ the volume and the form factor of a single scattering centre, respectively. The exchanged wave vector $Q$ is defined as

$$
Q=\frac{4 \pi}{\lambda} \sin \theta
$$

$2 \theta$ being the full scattering angle. Once the SDF $N(R)$ from (1) is known (Gerold 1961; Fedorova and Sthmidt 1978; Glatter 1980; Re Fiorentin 1982; Magnani et al 1988; Potton et al 1988), important microstructural parameters of the scattering centres, such as their density, specific surface, volume fraction and average dimension, can be easily calculated. During the study of the implanted materials, the asymptotic behaviour of the scattering cross section for large $Q$ (proportional to $Q^{-4}$ ) played an important role.

\section{Helium bubbles in metals}

The knowledge of the bubble parameters, such as density, average size and SDF, as a function of annealing time and temperature is of great interest to understand the mechanisms leading to the degradation of the mechanical properties in helium containing materials. It was decided to use SANS mainly because it gives quantitative 


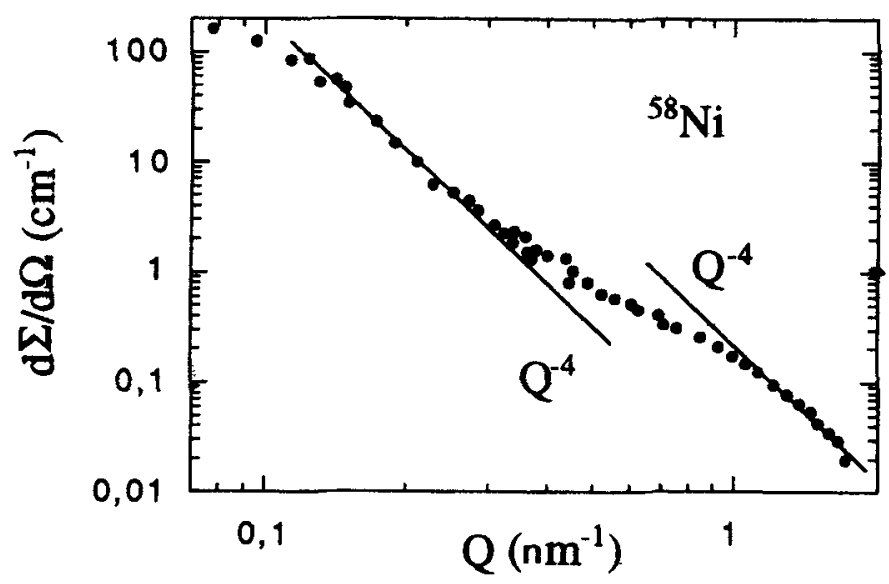

Figure 1. SANS cross section of the ${ }^{58} \mathrm{Ni}$ sample implanted at room temperature with 1200 appm helium and annealed at $1123 \mathrm{~K}$ for $2 \mathrm{~h}$.

results averaged over a macroscopic volume and because the contrast variation technique can provide information on the gas density inside the bubbles, impossible to obtain by TEM. The first important result obtained by SANS was to show a bimodal distribution of the scattering centres-helium bubbles-as it can be seen in figure 1 where a double asymptotic behaviour was found in the SANS cross section of the ${ }^{58} \mathrm{Ni}$ sample implanted with 1200 appm $\mathrm{He}$ and annealed at $1123 \mathrm{~K}$ for $2 \mathrm{~h}$ (Schwahn et al 1983). The sample was implanted at room temperature and subsequently isochronally annealed for $2 \mathrm{~h}$ at temperatures between 773 and $1123 \mathrm{~K}$. SANS measurements were performed after each annealing step, whereas TEM observations took place only after the last annealing at $1123 \mathrm{~K}$. The SANS data were fitted by using a spherical form factor and two log-normal SDFs were found centred around 1.4 and $13 \mathrm{~nm}$, respectively. On the other hand, TEM observations did not show any sign of large bubbles. Moreover, under the assumption of thermodynamic equilibrium bubbles, the total content of helium was found to be about 910 appm, i.e. below the measured value obtained by outgassing a small area from the centre of the implanted specimen (1200 appm), in excellent agreement with the nominal value. In order to confirm the presence of large bubbles, a ${ }^{58} \mathrm{Ni}$ specimen was implanted with 1550 appm helium ąnd subsequently isothermally annealed at $1073 \mathrm{~K}$ for $2,6.75$ and $50 \mathrm{~h}$.

SANS measurements were performed after each annealing step and TEM observations only after the last one (Kesternich et al 1984). In order to let helium bubbles coarsen, a large amount of vacancies has to be supplied. In the bulk there are not enough vacancies to allow the bubbles to reach dimension of the order of $10 \mathrm{~nm}$. Therefore, TEM observations were performed in the region neat the free surface. By covering one side of the TEM specimen before jet-polishing it, it was possible to obtain an electron transparent area near the surface.

In this way, large bubbles with mean radius of $8 \cdot 3 \mathrm{~nm}$ were found and the SDFs obtained by SANS and by TEM after the last annealing step, as reported in figure 2, are in good agreement. Causes of non perfect agreement between the SDFs are the lower resolution limit of the TEM technique (about $0.7 \mathrm{~nm}$ ) and the a-priori assumed log-normal shape of the SDF for the SANS data analysis. The large bubbles grow 


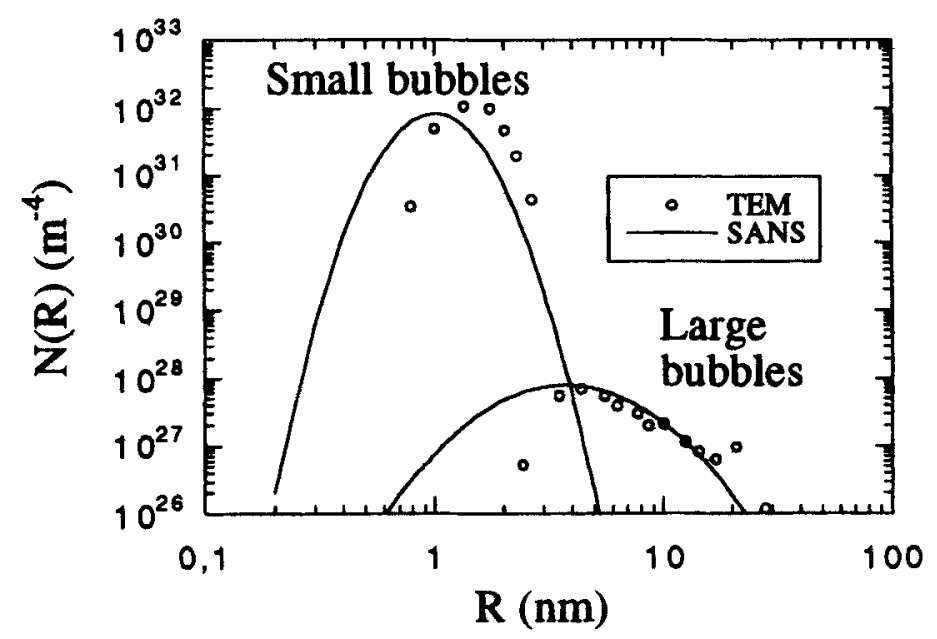

Figure 2. Size distribution functions of helium bubbles in ${ }^{58} \mathrm{Ni}$ implanted at room temperature with 1550 appm helium and annealed at $1073 \mathrm{~K}$ for $50 \mathrm{~h}$ obtained by SANS and TEM.

because of the larger amount of vacancies available in the vicinity of grain boundaries and external surfaces. In the two different regions the bubbles are slightly different (in the grain boundaries the bubbles are smaller) and in the large family seen by SANS both the grain boundaries and surface bubbles are included. Contrast variation technique in SANS was also applied to determine the gas pressure inside the bubbles. The integral in (1) depends only on the bubble population, and it does not change by using different $\mathrm{Ni}$ isotopes or by implanting ${ }^{3} \mathrm{He}$ or ${ }^{4} \mathrm{He}$. By performing SANS measurements on two different samples, the SANS cross sections ratio depends only on the scattering contrast ratio $\alpha$, which is related to the atomic volume ratio $\omega$, defined as $V_{\mathrm{Ni}} / V_{\mathrm{He}}$.

$$
\alpha=\left(\frac{\mathrm{d} \Sigma}{\mathrm{d} \Omega_{1}}(Q) / \frac{\mathrm{d} \Sigma}{\mathrm{d} \Omega_{2}}(Q)\right)=\frac{(\Delta \rho)_{1}^{2}}{(\Delta \rho)_{2}^{2}}=\left[\left(1-\omega \frac{b_{\mathrm{Ni}}}{b_{\mathrm{He}}}\right)_{1} /\left(1-\omega \frac{b_{\mathrm{Ni}}}{b_{\mathrm{He}}}\right)_{2}\right]^{2},
$$

In Schwahn et al (1983), two $\mathrm{Ni}$ isotopes $\left({ }^{58} \mathrm{Ni}\right.$ and $\left.{ }^{60} \mathrm{Ni}\right)$ were implanted with ${ }^{4} \mathrm{He}$. In that unlucky case the dimension of the small bubbles were such that the scattering contrast in ${ }^{60} \mathrm{Ni}$ was very weak and the SANS cross section negligible. In order to obtain suitable SANS cross sections, it was decided to implant two ${ }^{58} \mathrm{Ni}$ samples at room temperature with 1200 appm ${ }^{3} \mathrm{He}$ and ${ }^{4} \mathrm{He}$ (Qiang-Li et al 1990). The specimens were isochronally annealed for $2 \mathrm{~h}$ at temperatures between 673 and $1173 \mathrm{~K}$. As usual, after each annealing step SANS measurements were performed and after the last step also TEM observations were carried out. From the SANS cross section, $\alpha$ values were calculated. At $673 \mathrm{~K} \propto$ was found constant around 1.0 showing practically no bubbles. For higher temperature values down to 0.6 were found. From these values, $\omega$, the atomic volume of helium and helium density were calculated by using (3). The results are shown in figure 3, together with the values expected for thermodynamic equilibrium bubbles calculated with a surface energy $\gamma=1.8 \mathrm{Nm}^{-1}$.

Within their uncertainties it is evident that the experimental points lie far above the predicted curve for equilibrium bubbles. By using these values the calculated helium content in the sample was found to be $1220 \pm 100 \mathrm{appm}$, in excellent agreement with 


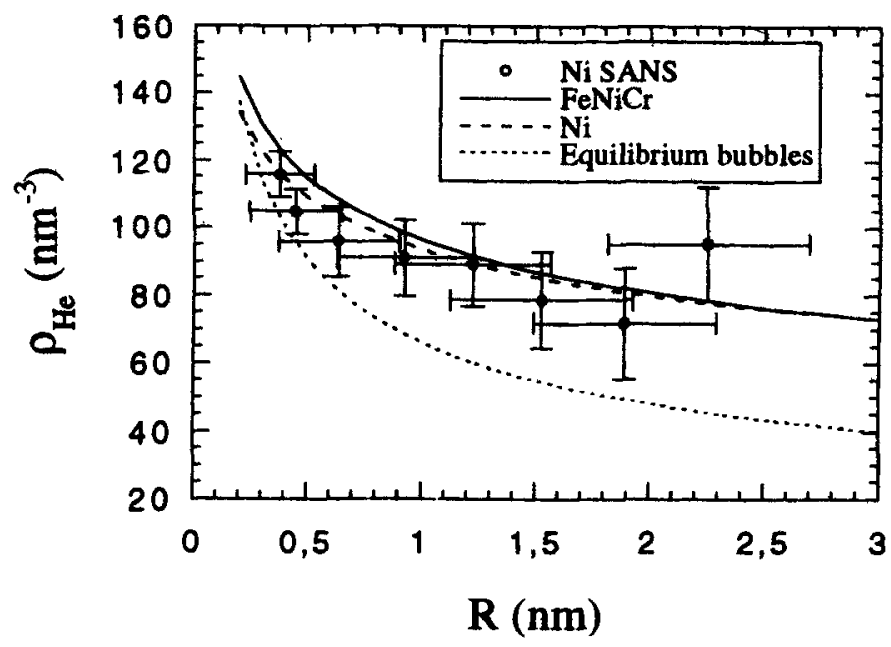

Figure 3. Helium density inside the bubbles as a function of the bubble radius. Experimental values (circles) and the best fit with a power law (dashed line) obtained with a contrast variation technique in ${ }^{58} \mathrm{Ni}$ implanted at room temperature with 1200 appm ${ }^{3} \mathrm{He}$ and ${ }^{4} \mathrm{He}$ and annealed at $1173 \mathrm{~K}$ for $2 \mathrm{~h}$, results obtained with a numerical procedure in $\mathrm{FeNiCr}$ implanted at room temperature with 1200 appm helium and annealed at $973 \mathrm{~K}$ (solid line) are compared to theoretical values for equilibrium bubbles calculated with a surface energy $\gamma=1.8 \mathrm{Nm}^{-1}$ (dotted line).

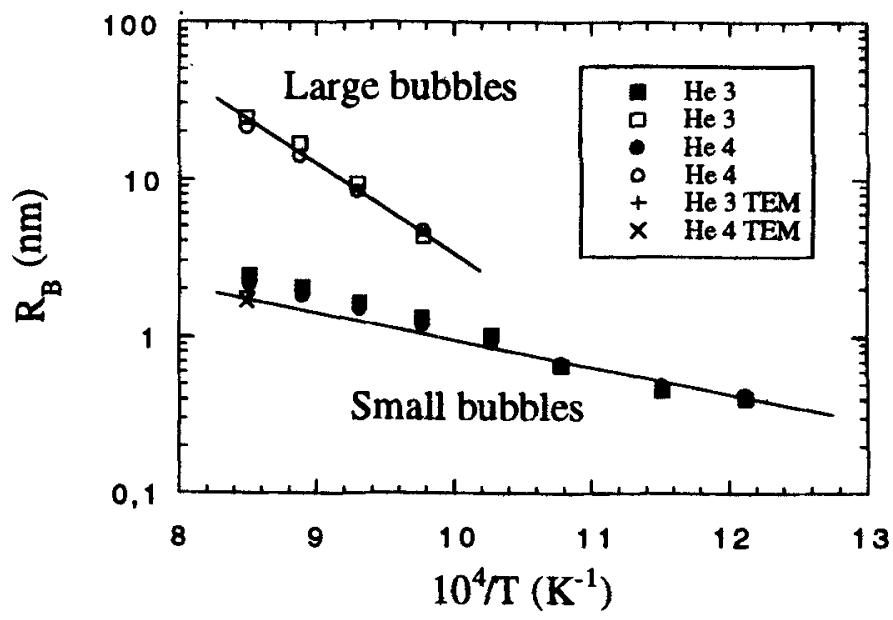

Figure 4. Mean radii of small and large bubbles in ${ }^{58} \mathrm{Ni}$ implanted at room temperature with 1200 appm of ${ }^{3} \mathrm{He}$ (squares) and ${ }^{4} \mathrm{He}$ (circles) and isochronally annealed at $1173 \mathrm{~K}$ for $2 \mathrm{~h}$ obtained from SANS data for temperatures between 823 and $1173 \mathrm{~K}$; TEM values of small bubbles for the last annealed step are also reported (crosses).

the nominal value. The gas pressure was also calculated via EOS (Trinkaus 1983) and the results show a constant overpressure of $3 \mathrm{GPa}$ compared to the equilibrium ones. The experimental gas density values were found to be well represented by a power law, as reported in figure 3. Large bubbles were found in the SANS data starting from $1023 \mathrm{~K}$ and in figure 4 are reported the average radii as a function of the reciprocal 
temperature for small and large bubbles for temperatures above $823 \mathrm{~K}$, the first temperature at which the presence of bubbles is revealed; a comparison with TEM data is also shown for the temperature of $1173 \mathrm{~K}$. The data are split into two branches, large and small bubbles, approximated by straight lines with different slopes in the Arrhenius-type plot. The activation energies of the coarsening processes were calculated from the slopes and 0.35 and $1.1 \mathrm{eV}$ were found for the small and large bubbles, respectively; these can be associated to low activation energy mechanism, such as migration and coalescence, for small bubbles and to high activation energy mechanism, such as Ostwald ripening, for large bubbles.

The effects of isothermal annealings at $973 \mathrm{~K}$ for times ranging from 0.28 up to $2780 \mathrm{~h}$ in the ternary alloy $\mathrm{Fe}-\mathrm{Ni}(15 \% \mathrm{wt})-\mathrm{Cr}(15 \% \mathrm{wt})$ implanted at room temperature with 1200 appm helium were investigated (Carsughi et al 1992). The material was choosen as a model alloy for the austenitic stainless steel; moreover, a direct comparison with the results obtained on nickel is possible, since their structures are very close to each other. The experimental SANS cross sections show no presence of bubbles before the end of the second annealing step $(2 \cdot 8 \mathrm{~h})$ and of the asymptotic
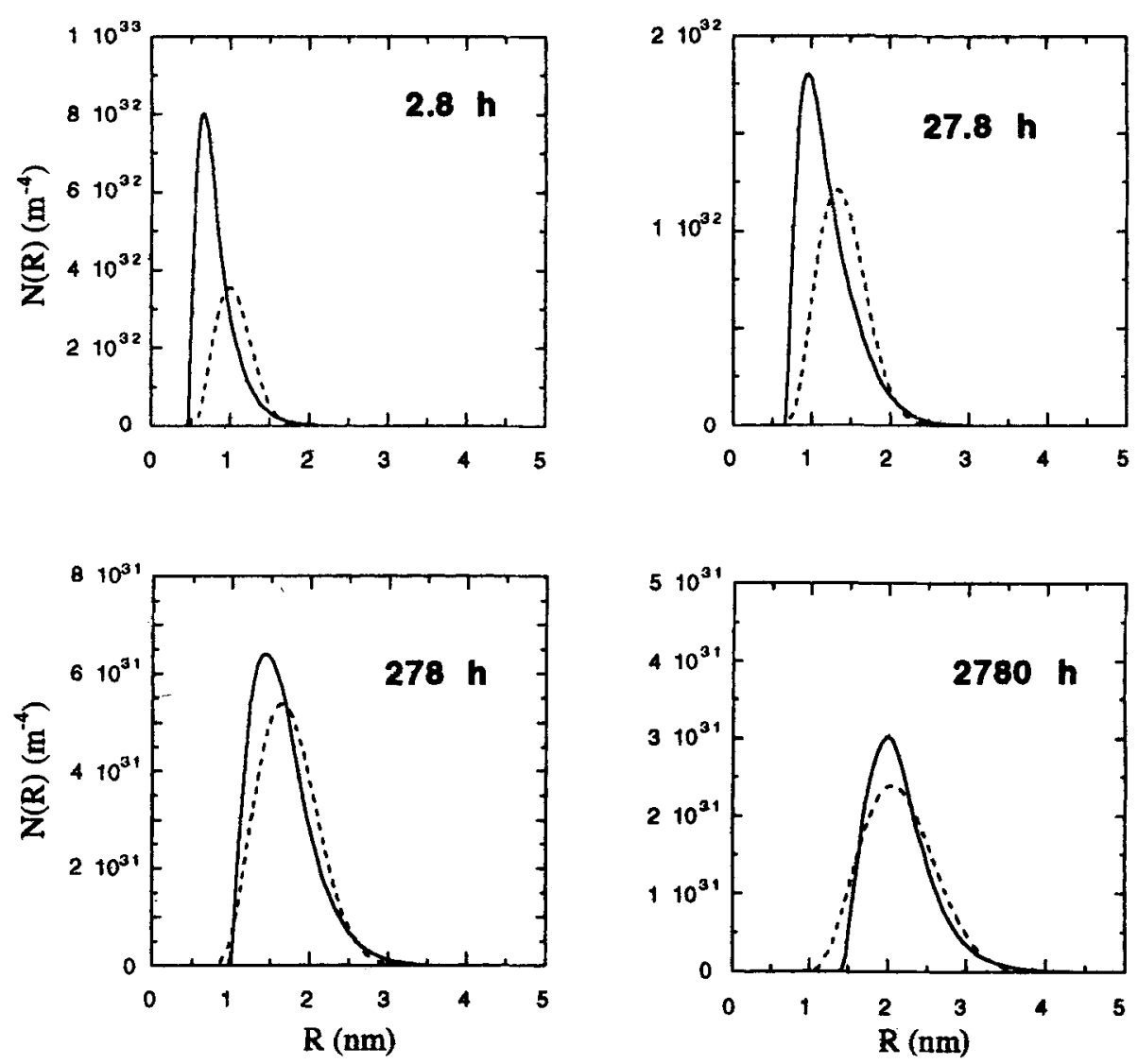

Figure 5. Bubble size distributions of small bubbles obtained by SANS (solid line) and by computer simulation of coarsening governed by migration and coalescence via surface diffusion (dashed line) in a $\mathrm{Fe}-\mathrm{Ni}(15 \% \mathrm{wt})-\mathrm{Cr}(15 \% \mathrm{wt})$ implanted with 1200 appm helium and annealed at $973 \mathrm{~K}$ for times between 2.8 and $2780 \mathrm{~h}$. 
behaviour of the large bubbles, probably due to the low annealing temperature used in this investigation. During the SANS data analysis the SDF was not choosen with an a-priori shape, but it was given by a linear combination of $\beta$-spline functions (Magnani et al 1988). The gas density inside the bubbles was obtained without contrast variation but the assumption that the content of helium remains constant during the whole annealing process was used. The gas density parameters of the power law were fitted and the result is shown in figure 3. Both the experimental results on nickel and on the ternary alloy are in excellent agreement with each other. The mean radii of the SDF as a function of annealing time can be approximated by a power law whose exponent is found to be $0 \cdot 14 \sim \frac{1}{7}$. The theoretical values predicted for this exponent are all larger than that found here, and, since the smallest one is $\frac{1}{6} \sim 0.17$ (Goodhew and Tyler 1981), it was reasonably assumed that the operating coarsening mechanism is migration and coalescence via surface diffusion.

The SDFs were also reproduced by computer simulation under the assumption of the same coarsening process (Carsughi et al 1994). A comparison of the SDFs of the small bubbles obtained by the experimental SANS data with those obtained from the simulation code is shown in figure 5. The agreement between both the SDFs becomes better with increasing annealing time, but can be considered satisfactory for all cases. The surface diffusion coefficient of $1 \cdot 10^{-18} \mathrm{~m}^{2} \mathrm{~s}^{-1}$ was calculated from the SANS data; within the usual range of uncertainty, it was found to be in good agreement with the values found in the literature. However, in the last work SANS data have not shown the presence of bubbles at grain boundaries; this was probably due to their low volume fraction, which is estimated to be less than $3 \%$, to the low annealing temperature and to the investigated $Q$ range. On the other hand, with TEM, they are evidenced as shown in figure 6.

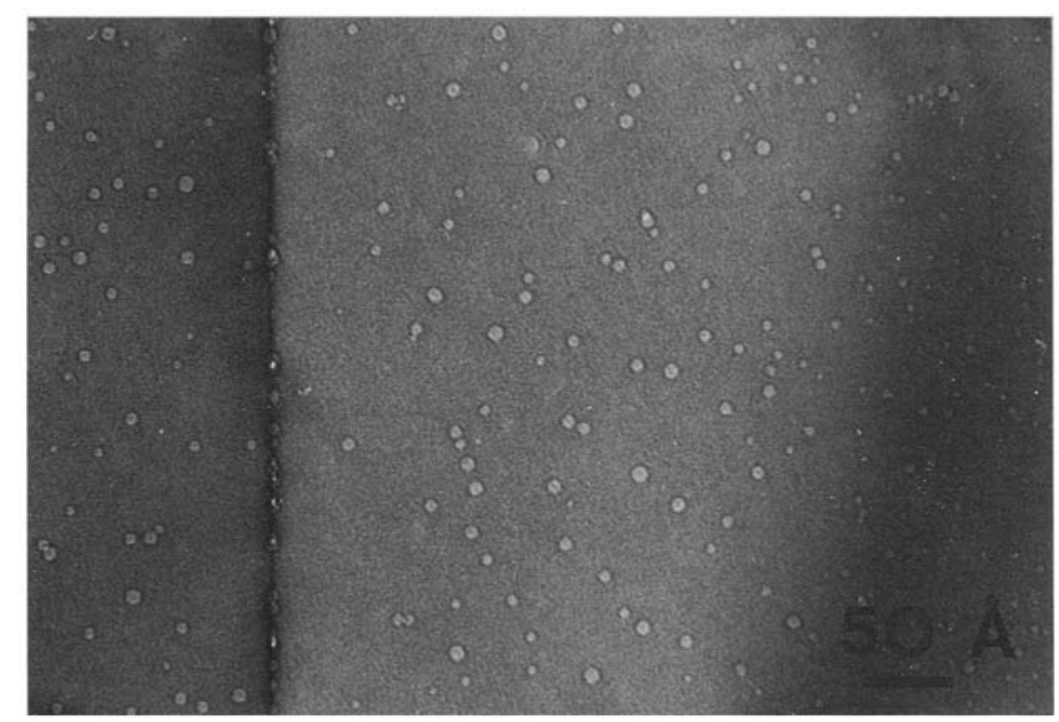

Figure 6. TEM micrograph of the helium bubbles in the vicinity of a grain boundary in $\mathrm{Fe}-\mathrm{Ni}(15 \% \mathrm{wt})-\mathrm{Cr}(15 \% \mathrm{wt})$ implanted at room temperature with 1200 appm helium and annealed at $973 \mathrm{~K}$ for $2780 \mathrm{~h}$. 


\section{Conclusions}

SANS is a powerful technique for investigating defects in condensed media. It produces quantitative results in a non-destructive way and it is very sensitive to local compositional changes. One of the major advantage of SANS is to give results averaged over a large volume; on the other hand, TEM gives detailed local information over a small selected volume. SANS played an important role in investigating implanted materials, showing the bimodal distribution of the bubble population and allowing the determination of the gas pressure inside the bubbles.

SANS and TEM should not be considered competitive but complementary techniques because their combination will give more complete information on the system. SANS was also successfully used in investigating helium bubbles in more complex materials, such as the martensitic steel MANET (Albertini et al 1991, 1992).

\section{Acknowledgements}

All the works presented here has been carried out at the Institut für Festkörperforschung of the Forschungszentrum Jülich. The author appreciates the close collaboration with Prof. Dr H Ullmaier, Dr W Kesternich, Dr H Schroeder, Dr D Schwahn, Dr $\mathrm{H}$ Trinkaus and Dr V Zell.

\section{References}

Albertini G, Carsughi F, Coppola R, Rustichelli F, Vlak W A H M and Van Dijk C $1991 \mathrm{~J}$. Nucl. Mater. 179-181 706

Albertini G, Carsughi F, Coppola R, Kesternich W, Mercurio G, Rustichelli F, Schwahn D and Ullmaier H 1992 J. Nucl. Mater. 191-1941327

Carsughi F, Kesternich W, Schwahn D, Ulimaier H and Schroeder H 1992 J. Nucl. Mater. 191-194 1284

Carsughi F, Ullmaier H, Trinkaus H, Kesternich W and Zell V 1994 J. Nucl. Mater. 212-215 336

Fedorova I S and Schmidt P W 1978 J. Appl. Cryst. 11405

Gerold V 1961 Phys. Status Solidi 137

Glatter O $1980 \mathrm{~J}$. Appl. Cryst. 137

Goodhew P J and Tyler S K 1981 Proc. R. Soc. London A377 151

Guinier $A$ and Fournet $G 1955$ Small angle scattering of $X$-rays (New York: John Wiley)

Kesternich W, Schwahn D and Ullmaier H 1984 Scr. Metall. 181011

Kostorz G (ed.) 1979 Treatise on material science and technology (New York: Academic Press ) 15 p. 227

Magnani M, Puliti P and Stefanon M 1988 Nucl. Instrum. \& Meth. A271 611

Potton J A, Daniel G J and Rainford B D 1988 J. Appl. Cryst. 21663

Price D L and Sköld K (eds) 1986 Methods of experimental physics (San Diego, Ca: Academic Press) 23A p. 1

Qiang-Li, Kesternich W, Schroeder H, Schwahn D and Ullmaier H 1990 Acta Metall. Mater; 382383

Re Fiorentin S 1982 J. Appl. Phys. 5353

Schwahn D, Ullmaier H, Schelten J and Kesternich W 1983 Acta Metall. 312003

Stuhrmann H B and Kirste R 1965 Z. f. Phys. 46247

Trinkaus H 1983 Rad. Eff. 78189

Ullmaier H 1981 Trans. Ind. Inst. Metals 34324

Ullmaier H 1984 Nucl. Fus. 241039

Ullmaier H 1991 Landolt-Börnstein new series III/25 (Berlin: Springer Verlag) p. 380 